\title{
SUR LES ORIGINES CELTES DE JOHN TOLAND
}

\author{
Alan HARrison
}

RÉsumé : Cet article établit que John Toland, né à la fin du xvII siècle, partageait la culture des Irlandais de langue maternelle gaélique. Il met en évidence l'intérêt de $\mathrm{J}$. Toland, tout au long de sa vie, pour la culture et les langues celtes et repère l'influence de celles-ci sur le contenu et la présentation de ses idées peu orthodoxes.

Mots clés : histoire, manuscrits, langues celtes, culture irlandaise.

SUMmaRY : This article establishes John Toland's background as an Irish speaker borm at the end of the seventeenth century. It traces his lifelong interest in the Celtic languages and their cultures and assesses the impact this had on the content and presentation of his unorthodox ideas.

Keywords : history, manuscripts, Celtic languages, Irish culture.

ZuSAMMENFASSUNG : Dieser Artikel versucht den linguistischen Hintergrund von John Toland als muttersprachlichem Sprecher des Irischen zu belegen. Er verfolgt dessen lebenslanges Interesse an den keltischen Sprachen und Kulturen und bewertet den Einfluß, den dieses Interesse auf den Inhalt und die Präsentation seiner unorthodoxen Ideen hatte.

Stichwörter : Geschichte, Manuskripte, keltische Sprachen, irische kultur.

Alan J. Harrison, né en 1943, M.A., Ph.D., est maître assistant d'irlandais moderne à l'University College de Dublin. Il étudie particulièrement le thêâtre médiéval et le xvır siècle irlandais.

Adresse: Department of Modern Irish Language and Literature, University College, Dublin 4, Ireland.

Revue de synthèse : $4^{\mathrm{e}} \mathrm{S} . \mathrm{N}^{\circ \mathrm{s}} 2-3$, avr.-sept. 1995, p. 345-355. 
Dans un ouvrage récent, où il suggère que John Toland fût la cible directe et indirecte de Tale of $a$ Tub de Jonathan Swift, Kenneth Craven se réfère à la formation celte de ces deux écrivains ${ }^{1}$. Cette affirmation, qui, dans le cas de J. Swift, n'est guère valable, est essentielle pour la compréhension de la position et du système intellectuels de Toland. Dans cette communication nous analyserons les relations de Toland avec la langue irlandaise ainsi que son travail dans le domaine des études celtes.

Il existe peu d'informations spécifiques sur la vie de Toland, mais ce qu'on sait de lui est bien connu ${ }^{2}$. Bien qu'il fût fidèle à ses origines et sût en faire profiter ses travaux intellectuels, on parle rarement du fait qu'il naquit et fut élevé dans une Irlande dont $90 \%$ de la population parlait le gaélique. Dans son History of the Druids, il prétendait que, pour lui, étudier les origines et les rites des druides allait de soi puisqu'il parlait le gaélique et avait été élevé dans l'extrême nord de l'Irlande, où il avait appris le vieux gaélique ${ }^{3}$. Il maintenait qu'une telle compétence linguistique était nécessaire à la compréhension des documents écrits en gaélique ainsi qu'à la connaissance d'autres langues celtes. Cette affirmation est d'extrême importance. Le degré d'alphabétisation, surtout en gaélique, dans la dernière moitié du XvII ${ }^{\mathrm{e}}$ siècle était très faible. Seuls les restes de l'ancienne classe dirigeante et quelques enthousiastes avaient une connaissance du gaélique écrit. Il n'existe pas de statistiques précises sur le degré d'alphabétisation chez ceux qui parlaient gaélique, mais nous estimons que moins de mille personnes savaient lire et écrire le gaélique, et ceci dans une Irlande dont un million de la population n'avait que le gaélique comme première langue ${ }^{4}$. C'est d'ailleurs pour cette raison que les tentatives de conversion de la population indigène au protestantisme, en traduisant les rituels de l'Église anglicane et d'autres littératures protestantes en gaélique, échouèrent.

1. Kenneth Craven, Jonathan Swift and the Millenium of Madness, Leyde/New York/ Cologne, Brill, 1992.

2. D.N.B.; A Collection of Several Pieces of Mr John Toland, éd. Pierre Des MaizeauX, Londres, 1726; Robert E. Sullivan, John Toland and the Deist Controversy, Cambridge, Mass./Londres, Harvard University Press, 1982; Stephen H. DanIEL, John Toland. His Methods, Manners and Mind, Kingston/Montreal, Mc Gill-Queen's University Press, 1984; Leslie STEPHEN, English Thought in the Eighteenth Century, Londres, 1876; J. Gerald Simms, "John Toland (1670-1722), a Donegal Heretic », Irish Historical Studies, XVI, 63, 1969, p. 304-320; Alan Harrison, «John Toland (1670-1722) and Celtic Studies », Proceedings of the Second North American Congress of Celtic Studies, Halifax, Nova Scotia, D'Arcy Mc Gee Chair of Irish Studies, St Mary's University, 1992, p. 653-679.

3. John Toland, The History of the Druids, Édimbourg, 1815, p. 55-56.

4. Kenneth H. Connell, The Population of Ireland, Oxford, 1950; Stuart Daultry, David Dickson et Cormac $O ́$ GrádA, "Eighteenth Century Irish Population : New Perspectives from Old Sources ", Journal of Economic History, 41, 3, 1981, p. 125-181. 
Le nom de famille irlandais à l'origine du nom « Toland » vient de «Uí Thuathalláin » (le petit-fils de Tuathallán) ${ }^{5}$. Ce nom est répandu dans les annales et généalogies, il est donc impossible de trouver l'aïeul éponyme de Toland. Cependant, d'après certaines informations les Uí Thuathallain étaient, par tradition, bardes ou historiens. Ceci implique une connaissance de la lecture et de la langue écrite. Toland naquit dans la dernière moitié du $\mathrm{XvII}^{e}$ siècle à l'époque de la disparition des écoles traditionnelles. Néanmoins, des vestiges de ces écoles auraient pu subsister encore dans sa région natale et, peut-être même, dans sa famille. Bref, ses connaissances du gaélique parlé et écrit se vérifient ici.

On ne peut pas identifier véritablement ceux qui encouragèrent les premières études de Toland; mais le fait qu'il reçut sa formation dans une université écossaise implique qu'il s'agissait d'apologistes presbytériens. L'influence exercée sur sa philosophie et sa politique pendant cette période en Écosse est bien connue et donc nous n'en parlerons pas ici. Notre propos est, en revanche, d'examiner les liens culturels entre l'Écosse et l'Irlande. Toland aurait rencontré des gens à Glasgow et à Édimbourg, qui parlaient le gaélique écossais (il existe une certaine intelligibilité mutuelle entre ces deux langues celtes) et $s$ 'intéressaient aux langues et aux vestiges manuscrits celtes. Au Moyen Âge, le monde gaélique comprenait l'Irlande entière, les îles et les Highlands de l'Écosse ainsi que l'île de $\operatorname{Man}^{6}$. En réalité, les Gaéliques écossais et irlandais ont leurs origines dans une seule langue mère datant de la première partie du xII siècle, le "Common Gaelic ». L'Irlande et l'Écosse partageaient beaucoup de traditions, dont celle d'une élite érudite, et jusqu'à la fin du XviI siècle, on sait que les bardes écossais se rendaient en Irlande et que les bardes irlandais se rendaient en Écosse. Ainsi, étant donné ses intérêts pour les documents anciens et ses compétences en gaélique, il est très probable que Toland ait rencontré d'autres savants dans ce domaine lors de son séjour en Écosse, comme, par exemple, Martin Martin qui écrivit sur les Western Isles ou encore le révérend John Beaton qui travaillait avec Edward Lhwyd, spécialiste reconnu des études celtes?

Au vrai, les premiers renseignements sur le travail de Toland dans ce domaine viennent d'Edward Lhwyd, un gallois né à Oswestery sur la frontière entre l'Angleterre et le Pays de Galles. Il fut nommé conservateur du musée Ashmolean à l'université d'Oxford en 1694. Il préparait une collection pour une étude comparative des pays celtes comprenant leurs langues. Ce travail fut publié dans son ouvrage Archaeologia Britannica en $1707^{8}$.

5. Edward Mac LySAGHT, The Surnames of Ireland, Dublin, Irish Academic Press, 1978.

6. Myles Dillon et Nora Chadwick, The Celtic Realms, Londres, Weidenfeld \& Nicolson, 1967.

7. John L. CAMPBell et Derek ThOMPSON, Edward Lhwyd in the Scottish Highlands, 16991700, Oxford, 1963.

8. Archaeologia Britannica, t. 1, Glossographia, Oxford, O.U.P., 1707. 
En 1694, avant l'arrivée de Toland à Oxford, E. Lhwyd écrivit à un ami pour lui dire qu'il essayait d'apprendre le gaélique irlandais mais qu'il ne trouvait personne à Oxford pour le lui apprendre?

Lorsque Toland vint à Oxford, après un séjour à Leyde puis à Utrecht, il était inévitable qu'il rencontrât E. Lhwyd. Pourtant leur rencontre n'eut pas une issue positive. En janvier 1694, E. Lhwyd écrivit :

"One Mr. Tholonne [sic] is lately come hither but as yet I am unacquainted with him with a design to write an Irish dictionary and a dissertation to prove the Irish a colony of the Gauls » ${ }^{10}$.

Une lettre écrite par Toland à cette époque montre qu'il comptait se servir de sa connaissance de la langue irlandaise pour entrer dans d'autres bibliothèques, en particulier celles qui possédaient des manuscrits bibliques, afin d'approfondir sa connaissance de l'hébreu ". Un lien intéressant existe entre les deux hommes : ils reçurent tous les deux le soutien et l'aide de John Mill, maître de Saint Edmond Hall et spécialiste des études bibliques. Il se peut même que ce soit J. Mill qui leur donna l'idée de faire une étude comparative des langues celtes, selon une approche qui devait faire d'E. Lhwyd le fondateur de la linguistique celte ${ }^{12}$. Toland ne se limita pas à la linguistique et il discuta longuement de politique et de philosophie dans les cafés et les bars. Son comportement amusa E. Lhwyd mais ne l'encouragea pas à collaborer avec l'homme d'Inishowen. Lorsque John Toland quitta Oxford, plus tard en 1694, E. Lhwyd écrivit à son propos : "[he is] one of excellent parts, but as little a share as may be of modesty or conscience; and one of the best scolds I ever met ${ }^{13}$.

Pour notre propos, il suffit de noter que Toland se servait de ses connaissances pour améliorer sa réputation et s'initier à l'étude comparative des langues celtes. Cette constatation semble évidente aujourd'hui, mais ne l'était pas à la fin du xvil ${ }^{e}$ siècle. Toland, comme Lhwyd, était l'un des pionniers dans ce domaine.

Tandis qu'il séjournait à Oxford, Toland avait probablement en chantier son Christianity not Mysterious. On sait maintenant que c'est d'avoir vu une esquisse de ce travail qui détermina John Locke à publier The Reasonableness of Christianity en 1695, avant l'ouvrage notoire de Toland ${ }^{14}$.

9. Robert T. GUNTHER, Life and Letters of Edward Lhwyd, Early Science in Oxford, vol. 14, Oxford, 1945, p. 249.

10. Ibid., p. 217.

11. A Collection of Several Pieces of Mr John Toland, op. cit. supra n. 2, t. II, p. 292-294.

12. Archaeologia Britannica, op. cit. supra n. 8, Préf.; J. Toland, op. cit. supra n. 3, p. 236.

13. R. T. GUNTHER, op. cit. supra n. 9, p. 278.

14. Voir S. Daniel, op. cit. supra n. 2; K. CRAVEN, op. cit. supra n. 1; et Justin Champion, The Pillars of Priestcraft Shaken: The Church of England and its Enemies, 1660-1730, Cambridge, Cambridge University Press, 1992, passim. 
De plus, à cause du scandale qui suivit la publication de Christianity not Mysterious, on ne parla pas de son intérêt pour les origines celtes, même quand on le retrouve plus tard en Europe. Pourtant, il faut noter qu'à cette époque, à Londres, il y avait beaucoup d'Irlandais, et surtout beaucoup de manuscrits irlandais dans diverses collections londoniennes. Par exemple, celle de John Bridges, duc de Chandos, comprenait la plupart des manuscrits collectionnés durant le XvII siècle par un amateur d'antiquités célèbre James Ware. D'autres manuscrits irlandais se trouvaient à la Royal Library, à la Cottonian Library et, plus tard, à la Harleian Library ${ }^{15}$. Quand Toland se rendit à Dublin en 1697, espérant recevoir un poste gouvernemental, il aurait eu accès non seulement à la bibliothèque de Trinity College mais aussi aux manuscrits des collections privées. D'après ce qu'on sait de lui, on peut être raisonnablement certain qu'il a profité de ces documents et qu'il en a intégré le contenu dans son bagage intellectuel. Ses connaissances semblaient d'ailleurs d'autant plus importantes que peu de gens étaient capables de mettre en question ses capacités en ce domaine.

De 1701 à 1710, Toland passa la plupart de son temps sur le continent, négociant, officiellement ou non, avec l'électrice de Hanovre au sujet de l'Act of Settlement. Il profita encore de la situation pour élargir ses relations intellectuelles. Deux de ses rencontres méritent d'être évoquées ici. En 1708, il se rendit à Prague où il demanda aux moines franciscains irlandais de lui fournir un certificat attestant qu'il était issu d'une vieille famille d'Inishowen, la presqu'île la plus au nord de l'Irlande ${ }^{16}$. Une étude des personnes qui fournirent ce document, deux O'Neills et un Devlin, confirme qu'il s'agissait non seulement de citoyens irlandais mais qu'ils étaient originaires de la même région et parlaient le gaélique. L'un de ces hommes, l'abbé Francis Devlin, après son retour en Irlande, eut beaucoup de contacts avec la coterie de scribes et de spécialistes du gaélique à Dublin ${ }^{17}$. Le certificat obtenu par Toland à Prague lui aurait permis de se faire accepter par les autres Irlandais en Europe, surtout par ceux des collèges irlandais dont les bibliothèques possédaient des manuscrits qui l'auraient intéressé. Ce qui est surprenant dans cette rencontre, c'est que l'antipathie des moines à l'égard de ce déiste, qui non seulement n'était pas catholique mais encore attaquait la religion à toute occasion, ne se manifesta apparemment pas, grâce aux sentiments de fraternité ressentis par des confrères gaéliques. Sauf erreur de notre part, il semble aussi que ses origines littéraires l'auraient aidé face aux lettrés irlandais en exil à Prague.

15. Robin Flower, Catalogue of Irish Manuscript in the British Museum, t. 2, Londres, 1926.

16. J. Toland, op. cit. supra n. 3, p. 2.

17. Alan Harrison, Ag Cruinniú Meala, Dublin, An Clóchomhar, 1988, passim. 
L'autre événement fut sa découverte d'un manuscrit qu'on appelle « Harle. 1802 » ou les « Gospels of Mael Brigte " ${ }^{18}$. Toland l'annonça dans Nazarenus, publié en 1718 , mais qui avait sa source dans des lettres écrites en Hollande en 1709. Deux lettres adressées à un protecteur important, que Toland appelle « Megaletor », et une introduction préliminaire ainsi qu'un appendice forment le corpus de ce livre. Le nom « Megaletor » et d'autres indices dans le texte nous donnent une idée de l'identité de son protecteur. $\mathrm{Ce}$ nom veut dire «celui à grand coeur" et on le trouve d'abord dans Oceana de James Harrington, édité par Toland et publié en 1700. Dans ce livre, le nom se réfêre au dirigeant idéal d'une république parfaite. Sans entrer dans les détails ici, nous tirons des indices trouvés dans l'ouvrage et son contexte immédiat, que ce « Megaletor » fut en réalité le prince Eugène de Savoie. Le prince Eugène, homme d'armes connu, vivait à cette époque en Hollande et fut une des lumières des sociétés secrètes de libres penseurs ${ }^{19}$. Il fut également un grand mécène et un amateur de livres. Dans la première lettre, Toland décrit un manuscrit en italien qui, selon lui, est une traduction de l'apocryphe Gospel of Barnabas. Il affirme aussi que cette «version » de l'écriture était acceptable pour les mahométans. Dans la préface ainsi que dans l'appendice, il nous informe que le prince est le véritable propriétaire de ce manuscrit. En réalité, nous pensons que Toland écrivit au prince au sujet de ce manuscrit et que ce dernier l'acheta par la suite.

La deuxième lettre de Nazarenus parle d'un manuscrit irlandais. Il s'agit d'une reproduction des Évangiles faite à Armagh en 1138 par un moine nommé Mael Brigte ${ }^{20}$. Le texte est essentiellement celui de la Vulgate. Il y a aussi divers commentaires et gloses en latin ainsi que des gloses, colophons et notes marginales en gaélique très difficiles à déchiffrer. Toland se sert des commentaires et du texte gaélique pour se lancer dans une analyse du christianisme irlandais originaire qui échappait, selon lui, au « priestcraft » et à la papauté. Il faut se rappeler ici qu'il lisait le gaélique, ce qui était déjà exceptionnel, et ajouter qu'il arrivait à déchiffrer le gaélique du $\mathrm{XII}^{\mathrm{e}}$ siècle, exploit encore plus exceptionnel. Ce phénomène était probablement plus rare que celui de savants anglais sachant lire les textes anglosaxons. John Toland se sert de ce texte pour suggérer que le mouvement «Culdee », dans les églises irlandaises et écossaises du vIII ${ }^{e}$ au $x^{e}$ siècle, montrait un christianisme non sacerdotal ${ }^{21}$.

18. ID., « John Toland and the Discovery of an Irish Manuscript in Holland », Irish University Review, t. 22, 1, 1992, p. 33-39.

19. Margaret C. JACOB, The Radical Enlightenment: Pantheists, Freemasons and Republicans, Londres, Allen and Unwin, 1981.

20. R. FLOWER, op. cit. supra n. 15, p. 428-432.

21. Peter O'DwYER, "Celtic Monks and the Culdee Reform », in An Introduction to Celtic Christianity, éd. James MACKEY, Édimbourg, T.\& T. Clark, 1989, p. 140-171. 
Il apparait que le manuscrit était l'un des manuscrits volés à la Bibliothèque royale de Paris par John Aymon, une « relation » de J. Toland à La Haye, qui est décrit comme « aventurier et prêtre renégat ${ }^{22}$. L'extrait suivant est tiré de la préface de Nazarenus et nous donne une idée du mélange d'érudition et de polémique de l'ouvrage :

"[...] be pleas'd to understand, that in the beginning of the same year, 1709, I discover'd at the Hague a manuscript of the four Gospels (then lately brought from France) all written in the Irish characters, which were mistaken for Anglosaxon, but yet the whole text in the Latin tongue. Some little thing in Irish itself is here and there mixt among the NOTES, which are very numerous, and other passages in the Irish language occur also else-where [...] I have set it in its true light, beyond what most others had an opportunity of doing, the Christianity originally profest in that nation [...] which appears to be extremely different from the religion of the present lrish ${ }^{23}$.

Les notes linguistiques et paléographiques de Toland sont très précises et montrent une grande familiarité avec le sujet. Pourtant, la plupart des spécialistes de l'histoire ancienne de l'église irlandaise ne seraient pas satisfaits de ses plaidoyers et de son emploi sélectif des faits. Sa thèse est un approfondissement de celle de James Usher, archevêque d'Armagh au milieu du XvII ${ }^{e}$ siècle. Elle plaisait (et plaît toujours) aux apologistes protestants, car elle leur permettait de voir la Réformation comme un retour aux anciennes valeurs et non comme un schisme. Toland avance l'idée que le christianisme de ce manuscrit représente une forme de républicanisme égalitaire et de liberté de conscience. Il est peu probable que ces traits correspondent à une Irlande monastique. Les compétences particulières en langues et en antiquités de Toland font partie ici de son arsenal polémique. Il est donc possible que la présentation de ce manuscrit ésotérique au prince Eugène lui ait concrètement permis d'améliorer sa réputation et de lui apporter une récompense matérielle pour son rôle d'intermédiaire dans les négociations pour l'achat de ce manuscrit. Néanmoins, lors de son retour à Londres, le « prêtre renégat » possédait toujours le manuscrit irlandais. Humfrey Wanley l'acheta finalement, avec d'autres manuscrits volés, pour la collection Harleian, probablement à l'instigation de Toland. Comme tant d'autres de ses ouvrages, Nazarenus fut attaqué et soutenu d'un égal élan; il est révélateur de noter que l'auteur rejette toute suggestion que quelqu'un puisse mettre en question sa maîtrise des sources irlandaises du texte ${ }^{24}$.

John Toland retourna à Londres en 1710 et y passa le reste de sa vie (à part un séjour supposé en Irlande en 1715). Son intérêt permanent pour

22. C. Ernest et Ruth C. WRIGHT, The Diary of Humphrey Wanley, 2 t., Londres, 1966, p. 439.

23. J. Toland, Nazarenus, Londres, 1718, p. x.

24. Par ex., in ID., Mangoneutes, Londres, 1720. 
les études celtes est manifeste dans trois publications de cette période : Nazarenus (1718), déjà évoqué; la traduction de Dermot O'Connor de la History of Ireland de Geoffrey Keating; et The History of the Druids, publié après sa mort.

Dermot O'Connor fut scribe du gaélique ainsi qu'artiste héraldique, et il fut employé par un prêtre irlandais, Anthony Raymond, pour traduire et interpréter des documents en gaélique. Raymond, un ami de Jonathan Swift, ancien chargé de cours à Trinity College de Dublin, s'intéressait énormément aux études irlandaises et était protecteur de scribes irlandais. En 1720, il eut un désaccord avec D. O'Connor et ce dernier partit pour l'Angleterre et emporta avec lui une idée de Raymond : celle de traduire et publier l'ouvrage de G. Keating. Ce livre, écrit vers 1630, était un modèle pour les apologistes gaéliques. Au début de 1721 , on retrouve D. O'Connor à Londres à la recherche d'un patronage pour la traduction : étant donné les circonstances, il est difficile d'imaginer qu'il ne rencontra pas Toland. La traduction, lors de sa publication au début de 1723, fut attaquée dans des livres, des annonces de journaux et dans des pamphlets et Toland se vit accusé d'être l'instigateur de l'entreprise ${ }^{25}$. On l'accuse plus précisément de promouvoir la mythologie et de s'en servir, afin d'avancer ses propres opinions panthéistes. Avec David Berman, nous avons discuté les faits et en tirons la conclusion, malgré la nature de ces preuves indirectes, qu'il est probable que Toland ait facilité et même influencé la publication de la traduction ${ }^{26}$.

The History of the Druids, comme Nazarenus, trouve sa source dans des lettres écrites auparavant à Robert Molesworth de 1718. L'ouvrage ne fut publié qu'en 1726 dans A Collection of Several Pieces of Mr John Toland par Pierre Des Maizeaux, quatre ans après la mort de Toland. Même si ce livre contient la version la plus complète du travail de Toland dans les études celtes, il faut remarquer que celui-ci l'aurait tenu pour incomplet, faute d'une préface écrite par lui-même. Nous n'analyserons pas les détails de ce travail ici et nous préférons laisser parler le texte lui-même.

John Toland fut un écrivain prolifique; il ressemble à un chercheur moderne dans la façon dont il amassa et accumula ses matériaux en les recyclant perpétuellement. À cause de l'intérêt porté à sa pensée, on néglige souvent son style et sa rhétorique. À tout moment, il aurait eu à sa disposition le matériel pour plus d'un livre et nous avons déjà remarqué la façon dont il se sert de son talent épistolaire pour écrire à ses protecteurs et

25. A. Harrison, op. cit. supra n. 17 , chap. 4.

26. David Berman et Alan Harrison, «John Toland and the Translation of Keating's History of Ireland », Donegal Annual, 36, 1984, p. 25-29. 
pour présenter sa philosophie. The History of the Druids est l'apogée de ses recherches et l'extrait suivant nous montre le peu de différences qui existent entre le livre et une lettre à Edward Lhwyd en 1694 :

"I presume to acquaint your Lordship with a design, which I form'd several years ago at Oxford, and which I have ever since kept in view [...] 'Tis to write the History of the Druids, containing an account of the ancient Celtic Religion and Literature [...] Tho' this be the subject, that will be naturally entertaining to the curious in every place; yet it does more particularly concern the inhabitants of antient Gaule, (now France, Flanders, the Alpine regions, and Lombardy) and all of the British Islands, whose antiquities are here partly explain'd and illustrated, partly vindicated and restor'd ${ }^{27}$.

Comme dans Nazarenus, les détails linguistiques et historiques sont mêlés à la rhétorique polémique. Ceci nous informe non seulement sur la culture celte mais aussi sur les thèses religieuses et politiques de Toland. Le critique moderne trouverait beaucoup à critiquer dans la présentation de Toland; cependant, son ouvrage est solide et impressionnant et montre bien plus qu'une connaissance éphémère de son sujet. La description qu'il fait des langues celtes reste valide et aurait sa place dans un ouvrage moderne sans avoir besoin de beaucoup de changements. Ses idées sur les origines et la nature des druides sont plus discutables. Ces thèses ont certainement leurs origines dans sa vision de l'histoire de la religion et servent à justifier son interrogation du christianisme traditionnel. L'intérêt qu'il portait à l'ancien christianisme celte découle, sans doute, de son désir d'établir la véracité de ses opinions. Dans un bel extrait, il anticipe les critiques de ses détracteurs et les retourne contre eux :

" but if in clearing up antient rites and customs, with the origin and institution of certain religious or civil societies (long since extinct), any communities or orders of men, now in being, should think themselves touched; they should not impute it to design in, the author, but to the conformity of things, if indeed there be any real resemblance: and, in case there be none at all, they should not make people apt to suspect there is, by crying out tho' they are not hurt. I remember, when complaint was made against an honorable person that, intreating of the Heathen Priests, he had whipt some Christian Priests on their backs; all the answer he made, was only asking, What made them get up there? The benefit of which answer I claim beforehand to myself, without making or needing any other apology. Yet if the correspondence of any Priests with heaven be as slenderly grounded as that of the Druids, if their miracles be as fictitious and fraudulant, if their love of riches be as immoderate, if their thirst after power be as insatiable, and their exercise of it be as partial and

27. J. Toland, op. cit. supra n. 3, p. 43. 
tyrannical over the Laity: then, I am not only content they should be touched, whether I thought of them or not; but, that they should be blasted too, without the possibility of ever sprouting up again. For truth will but shine the brighter, the better its counterfeits are shewn ${ }^{28}$.

Il ne s'agit nullement ici d'une recherche objective de la vérité mais plutôt de l'utilisation par Toland de ses connaissances, afin de promouvoir et de défendre, contre la religion institutionnalisée, les thèses qu'il soutenait depuis longtemps déjà. Un autre extrait nous montre comment ses propres affaires exercent une influence sur sa polémique :

«Dr. Kennedy says, that Patric burnt 800 volumns, stuft with the fables and superstitions of Heathen Idolatry: unfit, adds he, to be transmitted to posterity. But pray how so? why are Gallic or Irish superstitions more unfit to be transmitted to posterity, than those of the Greecs and Romans? Why shou'd Patric be more squeamish in this respect than Moses or the succeeding Jewish Prophets, who have transmitted to all ages the Idolatries of the Egyptians, Phenicians, Caldeans, and other Eastern nations? What an irreparable destruction of history, what a deplorable extinction of arts and inventions, what an unspeakable detriment to Learning, what a dishonor upon human understanding, has the cowardly proceeding of the ignorant, or rather of the interested, against unarm'd monuments at all times occasion'd! And yet this bookburning and letter-murdring honor, tho' far from being commanded by Christ, has prevail'd in Christianity from the beginning : As in the Acts of the Apostles we read that many of them which believ'd - and us'd curious arts, brought their books together, and burnt them before all men; and they counted the price of them and found it fifty thousand pieces of silver, or about three hundred pounds sterling. This was the first instance of burning books among Christians; and ever since that time the example has been better follow'd than any precept of the Gospel ${ }^{29}$.

Bien qu'il ne l'explicite pas clairement ici, il n'est pas douteux que cet extrait soit une réaction à la mise à feu publique de son Christianity not Mysterious à Dublin en 1697. Mais ses contemporains auraient bien compris toutes les nuances du texte.

Outre les commentaires et l'analyse linguistique des vieux manuscrits, il existe une autre facette novatrice de The History of the Druids qui donne à l'ouvrage un aspect très moderne. Il s'agit de la manière dont il explique souvent ses arguments en donnant des exemples tirés de ses propres expériences. Un de ces extraits nous donne une des premières explications d'une vieille tradition saisonnière, celle des feux de Saint John's Eve :

"Thus I have seen the people running and leaping thro' th St. John's fires in Ireland, and not onely proud of passing unsing'd : but as if it were some kind

28. Ibid., p. 53-55.

29. Ibid., p. 93-94. 
of lustration, thinking themselves in a special manner blest by this ceremony, of whose original they were wholly ignorant in their imperfect imitation ${ }^{30}$.

De telles observations soulignent le fait que John Toland était un observateur pénétrant et donnent à son travail un certain charme.

Nous nous sommes limité ici au travail linguistique et historique de Toland mais il faudrait y ajouter ses observations sur l'archéologie des pays celtes. Elles figurent dans les deuxième et troisième lettres de The History of the Druids. Elles sont fondées principalement sur son expérience des sites in situ, sur une lecture de la littérature contemporaine et sur les histoires populaires sur les origines de ces ruines ${ }^{31}$. Il ne nous incombe pas de déterminer l'importance de son travail dans ce domaine, mais d'après des collègues archéologues, son travail dans ce domaine est également très intéressant.

En conclusion, il s'avère que John Toland était fondé à se réclamer de ses origines irlandaises et qu'il se distingua avec talent par sa connaissance du monde celte. Il existe des preuves attestant qu'il passa sa vie à étudier le gaélique irlandais ainsi que d'autres langues celtes. Dans les domaines de la culture et de la religion celtes, il avait bien exploré les textes anciens et contemporains. La plus grande part de son travail et de ses analyses sur les textes révèle une compétence remarquable; cependant, ses arguments concernant la religion celte et les druides sont plus téméraires que ceux de ses contemporains, et ne seraient pas acceptables pour les critiques modernes de ce domaine. Néanmoins, ses idées demeurent intéressantes et utiles. Ses opinions sur la religion et la politique changèrent peu et le matériel celte forma partie intégrante du corpus qui étaya sa vie intellectuelle et il s'en servit pour mettre en valeur ses positions tout au long de sa vie. Les spécialistes des études celtes n'estiment pas le travail de Toland à sa juste mesure. Mềme s'il n'est pas le savant le plus important dans ce domaine, il est difficile de l'ignorer si on veut comprendre l'histoire de leur développement. Inversement, les études celtes ne doivent pas être oubliées par ceux qui désirent mieux connaître John Toland.

30. Ibid., p. 112.

31. Ibid., Letters 2 et 3, p. 96-217. 